\title{
GPC Multivariable Control Applied to Temperature and Humidity Neonate Incubators
}

\section{A. Zermani}

Université de Tunis El Manar,

Ecole Nationale d'Ingénieurs

de Tunis, Laboratoire de recherche

Analyse et Commande des Systèmes, 1002, Tunis, Tunisie,

aymen_net@voila.fr
E. Feki

Université de Tunis El Manar,

Ecole Nationale d'Ingénieurs

de Tunis, Laboratoire de recherche

Analyse et Commande des Systèmes,

1002, Tunis, Tunisie,

elyes.feki@istmt.rnu.tn

\author{
A. Mami \\ Université de Tunis El Manar, \\ Ecole Nationale d'Ingénieurs \\ de Tunis, Laboratoire de recherche \\ Analyse et Commande des Systèmes, \\ 1002, Tunis, Tunisie, \\ mami.abdelkader@planet.tn
}

\begin{abstract}
This paper presents a multivariable predictive control algorithm to resolve the coupling effect in closed loop systems. The desired decoupling is achieved by including an appropriate weighting factor on the objective function of predictive control. This weighting factor is tuned in synchronization to the error observation to eliminate the strong interaction. The proposed algorithm is applied to the control of humidity and temperature of a neonatal incubator. Simulation results are verified and compared with the others algorithm in the literature.
\end{abstract}

Keywords- predictive control; decoupling; weight factor; incubator

\section{INTRODUCTION}

For many years, incubators are used to produce healthful micro-environment in order to reduce new born heat loss. Temperature and humidity are the important factors that need to be maintained with a minimum variation. Furthermore, the current commercial incubator system ensures the control of the temperature and use a passive humidification system for humidity without control [1]. This method cannot provide a high humidity level at low temperature such as in the range of 23-38 degree. For this reason, we have developed the humidification system based on a nebulizer $[2,3]$.

The decentralized method of designing multivariable control system is one of the most popular control strategies used in practice. $[4,5,6]$. In the sequential algorithm, the Multi-Input Multi-Output model (MIMO) is decomposed into a sequence of single variable (SISO) so that only one control loop SISO is tuned at a time. The advantages of this type of project are well reported in literature $[4,5,7,8]$. In this article, an experiment method is proposed to determine the transfer function matrix of the incubator system, which decouples the Two-Input Two -Output (TITO) system into four independent loops. This method produced a discrete-time system transfer matrix whose parameters were estimated in real time by the extended least-squares algorithm.

Generalized predictive control appears to be attractive solution in multivariable process control [9, 10]. However, these controllers cannot solve problems with strong interaction. To resolve this problem we focused on the development of a decentralized predictive decoupling controller. Our proposed decoupling method is achieved by including an appropriate weighting factor on the objective function of predictive control and compared with other algorithm in the literature [11].

The paper is structured as follows: The section 2 is devoted to present incubator description, modeling and validation. In section 3 , first we present the decentralized generalized predictive control with constraint next, we developed the method of decoupling based on adding an error weighting factor. In section 4, computer simulations are conducted shown the advantages of the proposed algorithm to decrease the coupling effect between controlled variables (temperature and humidity) of incubator system, and compared to others proposed in the literature. Finally, the conclusion is drawn in section 5 .

\section{INCUBATOR SYSTEM}

\section{A. Description}

The incubator consists basically of two divisions figure1: the first division is a small (approx- imately: $0.5 \times 0.5 \times 1 \mathrm{~m}^{3}$ ) cabinet with transparent walls so that the infant can be easily observed. The device may include an AC-powered heater, a fan to circulate the warmed air. The second division is an humidification chamber in Plexiglas based on a ultrasonic nebulizer, which is an instrument for converting a liquid into a fine spray. This system is able to increase the humidity to $80 \%$.

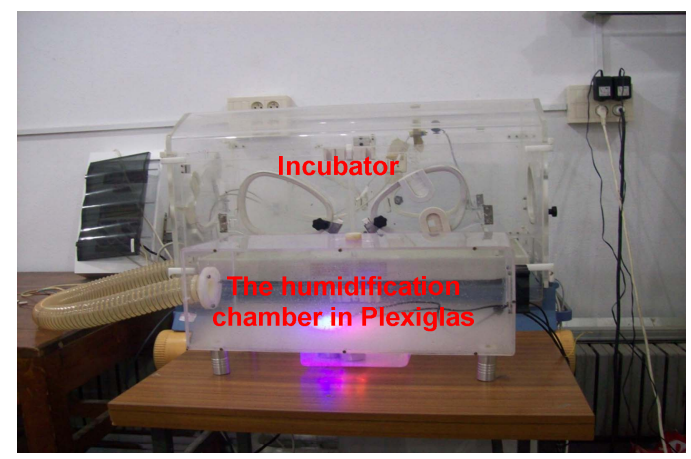

Fig. 1. Schematic illustration of the experimental unit's 
Also we developed a microcontroller-based system devoted to control the humidity and the heating of the newborn incubator. The system developed measures the air temperature and the humidity by tow sensors LM35 and SY-230 [12]. Then, these data were exported to a microcomputer to be analyzed.

\section{B. Modeling}

Identification process of incubator is the first step for design and implementation controller. An experiment method is proposed to determine the transfer function matrix, which decouples the TITO system into four independent loops.

The transfer function matrix of the incubator system can be expressed as follow:

$$
\left[\begin{array}{c}
Y_{1}(k) \\
Y_{2}(k)
\end{array}\right]=\left[\begin{array}{ll}
G_{11}(z) & G_{12}(z) \\
G_{21}(z) & G_{22}(z)
\end{array}\right]\left[\begin{array}{c}
U_{1}(k) \\
U_{2}(k)
\end{array}\right]+\left[\begin{array}{c}
e_{1}(k) \\
e_{2}(k)
\end{array}\right]
$$

The input-output relation of the system is shown in Fig.2

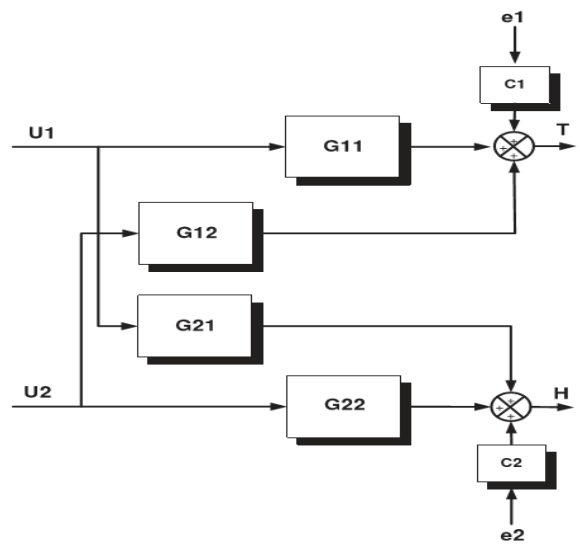

Fig. 2. Input-output model of incubator system.

The inputs are:

$U 1$ : control signal applied to the heater,

$U 2$ : control signal applied to the nebulizer.

The outputs are:

Y1: temperature value output signal $(\mathrm{T})$,

Y2: humidity level output signal $(\mathrm{H})$.

G11 is a transfer function showing the relation between input $\mathrm{U} 1$ and output $y 11$. Likewise, G12 is the transfer function that shows the effect of input $U 2$ to output $y 12, G 21$ indicates the effect of input $U 1$ to $y 21 ; G 22$ indicates the effect of input $U 2$ to output $y 22$.

The transfer functions of the various subsystems are described as follow:

$$
y_{i j}(k)=\frac{z^{-d i j} B_{i j}\left(z^{-1}\right)}{A_{i j}\left(z^{-1}\right)} U_{i}(k-1)
$$

$$
\begin{aligned}
& Y_{1}(k)=y_{11}(k)+y_{12}(k)+e_{1}(k) \\
& Y_{2}(k)=y_{22}(k)+y_{21}(k)+e_{2}(k)
\end{aligned}
$$

Where $i$ and $j$ are the level indices $i, j=\{1,2\}$ and $A_{i j}, B_{i j}$ and $C_{i j}$ are polynomials:

$$
\begin{aligned}
& A_{i j}\left(z^{-1}\right)=1+a_{1} z^{-1}+a_{2} z^{-2}+\cdots+a_{n a} z^{-n a} \\
& B_{i j}\left(z^{-1}\right)=b_{0}+b_{1} z^{-1}+b_{2} z^{-2}+\cdots+b_{n b} z^{-n b} \\
& C_{i j}\left(z^{-1}\right)=1+c_{1} z^{-1}+c_{2} z^{-2}+\cdots+c_{n c} z^{-n c}
\end{aligned}
$$

The transfer functions were obtained by means of experimental tests through the input, the output data collection and the later numeric treatment of this information. For multivariable open loop experiments, the usual practice is to apply mutually independent pseudo-random binary signals (PRBSs) to all the manipulated variables of the plant.

To find $G 11(z)$ an input $U 2=0$ is applied and the relation between input $U 1$ and output $Y 1=y 11$ is found.

An equivalent equation in differential form can be written:

$$
\begin{aligned}
Y_{l}(k)= & -a_{1} Y_{l}(k-1)-a_{2} Y_{l}(k-2)+b_{1} U_{1}(k-d-1)+b_{2} U_{I}(k-d-2) \\
& +c_{1} \xi_{1}(k-1)+c_{2} \xi_{I}(k-2)
\end{aligned}
$$

The output equation can be obtained as below:

$$
\begin{aligned}
& Y_{I}=\theta_{I} f_{1} \\
& \theta_{I}=\left[\begin{array}{llllll}
-a_{I} & -a_{2} & b_{2} & b_{2} & c_{1} & c_{2}
\end{array}\right] \\
& f_{I}=\left[\begin{array}{llllll}
Y_{I}(k-1) & Y_{I}(k-2) & U_{I}(k-1-d) & U_{I}(k-2-d) & \xi_{I}(k-1) & \xi_{I}(k-2)
\end{array}\right]^{T}
\end{aligned}
$$

The Least squares algorithm is as follow:

$$
\begin{aligned}
& P(k)=\frac{1}{\lambda_{1}}\left[P(k-1)-\frac{P(k-1) \varphi_{i j}(k) \varphi_{i j}{ }^{T}(k) P(k-1)}{\frac{\lambda_{1}}{\lambda_{2}}+\varphi_{i j}{ }^{T}(k) P(k-1) \varphi_{i j}(k)}\right] \\
& K(k)=P(k) \varphi_{i j}(k) \\
& \theta_{i j}(k)=\theta_{i j}(k-1)+K(k)\left(y_{i j}(k)-\varphi_{i j}(k)^{T} \theta_{i j}(k-1)\right)
\end{aligned}
$$

To found $G 12(z), G 21(z)$ and $G 22(z)$, a same calculation of $G 11(z)$ are used.

At the end of the experiments, the matrix elements of the system transfer function are :

$$
G(z)=\left[\begin{array}{cc}
\frac{5.8278 e-005 z^{-3}+9.3029 e-004 z^{-4}}{1-0.5962 z^{-1}-0.3984 z^{-2}} & 0 \\
\frac{2.392 e-005 z^{-1}+3.622 e-005 z^{-2}}{1-1.981 z^{-1}--0.9803 z^{-2}} & \frac{0.00203 z^{-3}+0.00088 z^{-4}}{1-0.5091 z^{-1}-0.4262 z^{-2}}
\end{array}\right]
$$

\section{Validation}

The model validation is performed by comparing the simulation results with real results from the incubator. 


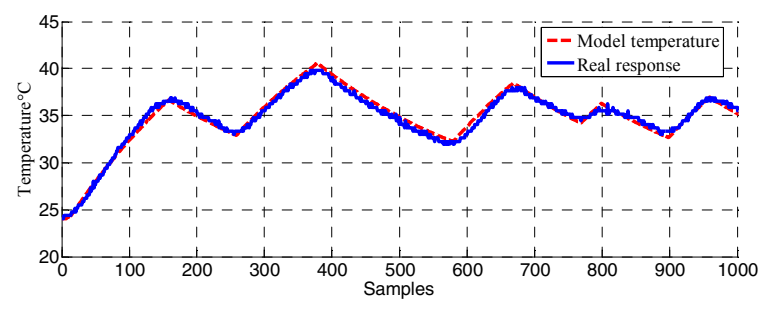

Fig. 3. Real and estimated temperature $\mathrm{G}_{11}$.

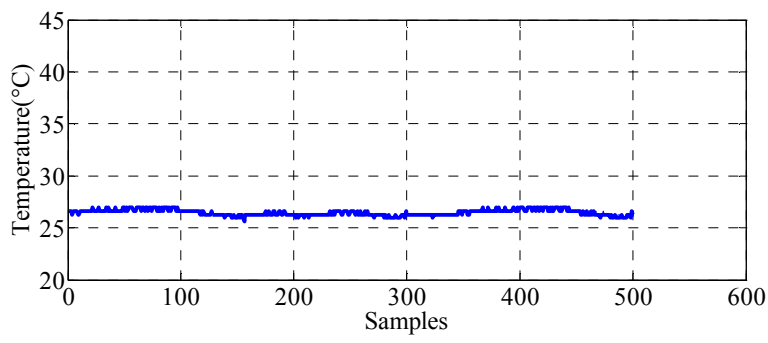

Fig. 4. Real temperature $\mathrm{G}_{12}$.

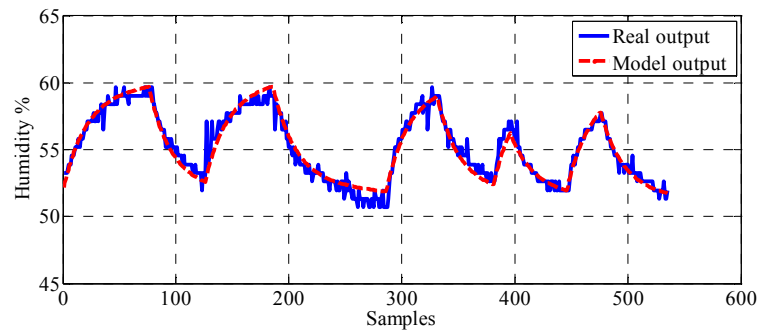

Fig. 5. Real and estimated humidity $\mathrm{G}_{22}$.

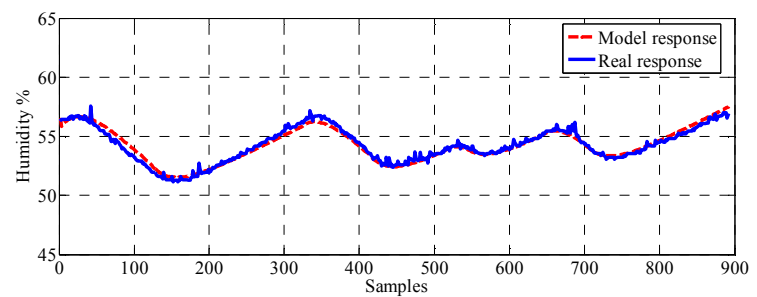

Fig. 6. Real and estimated humidity $\mathrm{G}_{21}$.

$$
V A R=\max \left\{1-\frac{\operatorname{var}(y-\hat{y})}{\operatorname{var}(y)}, 0\right\} * 100 \%
$$

\begin{tabular}{|c|c|c|c|}
\hline VALIDATION & G11 & G22 & G21 \\
\hline$V A R$ & $94.089 \%$ & $90.604 \%$ & 97.7574 \\
\hline
\end{tabular}

As it is clear from the structure of the identified model, the temperature variation is almost not related to the change in moisture level $(\mathrm{G} 12=0)$, and this weak relation can be modeled as a disturbance to the system. The other components are simply modeled with a second order system with a time varying delay. This structure of the model can be used as a start up model for an adaptive GPC synthesis as future work.

\section{DECOUPLING PREDICTIVE CONTROL}

\section{A. Cost Function}

The generalized predictive control based on the minimization of a quadratic criterion on a sliding horizon, which involves a term related to the difference between the predicted output sequence and the sequence of future control [11]. The criterion is given by the following relation:

$J_{i}=\lambda_{y i} \sum_{t=N_{i}}^{H P_{i}}\left[y_{C_{i}}(k+t)-\hat{y}_{i}(k+t)\right]^{2}+\lambda_{u i} \sum_{t=1}^{N_{C_{i}}} \Delta u_{i}^{2}(k+t-1)$

With:

- $\hat{\mathrm{y}}_{\mathrm{i}}(\mathrm{k})$ : the output value predicted at time $k$,

- $\mathrm{y}_{\mathrm{C}_{\mathrm{i}}}(\mathrm{k})$ : the set points values at time $k$,

- $\Delta \mathrm{u}_{\mathrm{i}}(\mathrm{k})$ : the increment of control at time $k$,

$-\mathrm{N}_{\mathrm{i}}$ : The minimum prediction horizon,

- $\mathrm{HP}_{\mathrm{i}}$ : The maximum prediction horizon,

$-\mathrm{N}_{\mathrm{C}_{\mathrm{i}}}$ : The control horizon,

$-\lambda_{\text {ui }}$ : The control-weighting factor.

$-\lambda_{\mathrm{yi}}$ : The error weighting factor.

\section{B. Prediction of the System Output}

The approach of generalized predictive control is based on a dynamic model of type ARIMAX (Auto-Regressive Integrated Moving Average with eXogenous input), given by the following form:

$$
\begin{aligned}
& A_{i}\left(z^{-1}\right) y_{i}(k)=z^{-d_{i}} B_{i}\left(z^{-1}\right) u_{i}(k-1)+C_{i}\left(z^{-1}\right) \frac{\xi_{i}(k)}{\Delta\left(z^{-1}\right)} \\
& y_{F_{i}}(k)=F_{i}\left(z^{-1}\right) y_{i}(k)
\end{aligned}
$$

With $\Delta\left(z^{-1}\right)=1-z^{-1}$ corresponds to an integral action.

Using (12), the output at time $(k+t)$ will be:

$$
y_{F_{i}}(k+t)=\frac{F_{i}\left(z^{-1}\right) B_{i}\left(z^{-1}\right)}{A_{i}\left(z^{-1}\right)} u_{i}\left(k+t-d_{i}-1\right)+\frac{F_{i}\left(z^{-1}\right) C_{i}\left(z^{-1}\right)}{A_{i}\left(z^{-1}\right) \Delta\left(z^{-1}\right)} \xi_{i}(k+t)
$$

By applying the Euclidean algorithm on the second term of (13), we get:

$$
\frac{C_{i}\left(z^{-1}\right)}{A_{i}\left(z^{-1}\right) \Delta\left(z^{-1}\right)}=L_{t}\left(z^{-1}\right)+z^{-1} \frac{G_{t}\left(z^{-1}\right)}{A_{i}\left(z^{-1}\right) \Delta\left(z^{-1}\right)}
$$

Using (13) and (14) and we assuming that the term related to the disturbance is zero, the optimal predictor of the output is written as follows:

$$
\hat{y}_{F_{i}}(k+t)=\frac{L_{t}\left(z^{-1}\right) F_{i}\left(z^{-1}\right) B_{i}\left(z^{-1}\right) \Delta\left(z^{-1}\right)}{C_{i}\left(z^{-1}\right)} u_{i}\left(k+t-d_{i}-1\right)+\frac{G_{t}\left(z^{-1}\right)}{C_{i}\left(z^{-1}\right)} y_{F i}(k)
$$

A second Diophantine equation decompose the predictor in two terms: a first term based on the current output, old orders, the system output and a second term dependent on future orders.

$$
\frac{\sigma_{i}\left(z^{-1}\right)}{C_{i}\left(z^{-1}\right)}=H_{t}\left(z^{-1}\right)+z^{-t+d} \frac{R_{t}\left(z^{-1}\right)}{C_{i}\left(z^{-1}\right)}
$$


With:

$$
\sigma_{i}\left(z^{-1}\right)=L_{t}\left(z^{-1}\right) F_{i}\left(z^{-1}\right) B_{i}\left(z^{-1}\right)
$$

The optimal predictor of the output is written as follows:

$\hat{y}_{F_{i}}(k+t)=H_{t}\left(z^{-1}\right) \Delta\left(z^{-1}\right) u_{i}\left(k+t-d_{i}-1\right)$

$$
+\frac{G_{t}\left(z^{-1}\right)}{C_{i}\left(z^{-1}\right)} y_{F i}(k)+\frac{R_{t}\left(z^{-1}\right)}{C_{i}\left(z^{-1}\right)} \Delta\left(z^{-1}\right) u_{i}(k-1)
$$

Where: $H_{t}\left(z^{-1}\right), G_{t}\left(z^{-1}\right), R_{t}\left(z^{-1}\right)$ et $L_{t}\left(z^{-1}\right)$ are polynomial solutions to the Diophantine equations [13].

The matrix formulation is represented as follows:

$$
\hat{Y}_{i}(k)=\hat{H} \Delta U_{i}(k)+\frac{\hat{G}(k) Y_{i}(k)}{C_{i}\left(z^{-1}\right)}+\frac{\hat{R} \Delta U_{i}(k-1)}{C_{i}\left(z^{-1}\right)}
$$

With:

$$
\begin{gathered}
\Delta U_{i}=\left[\Delta u_{i}(k) \cdots \Delta u_{i}\left(k+N_{C_{\mathrm{i}}}-1\right)\right]^{T} \\
\hat{G}=\left[G_{I+d}\left(z^{-1}\right) \cdots G_{H P_{i}+d_{\mathrm{i}}}\left(z^{-1}\right)\right]^{T} \\
\hat{R}=\left[R_{I+d_{i}}\left(z^{-1}\right) \cdots R_{H P_{i}+d_{i}}\left(z^{-1}\right)\right]^{T} \\
\hat{H}=\left(\begin{array}{cccc}
h_{0} & 0 & \cdots & 0 \\
h_{1} & h_{0} & \cdots & 0 \\
\vdots & \vdots & \vdots & \vdots \\
h_{H P_{i}-1} & h_{H P_{i}-2} & \cdots & h_{H P_{i}-N_{C i}}
\end{array}\right)
\end{gathered}
$$

\section{Control law}

We can write the criterion $J$ in matrix form:

$$
J_{i}=\left[\hat{Y}_{i}(k)-Y_{C_{i}}(k)\right]^{T} \chi_{i}\left[\hat{Y_{i}}(k)-Y_{C_{i}}(k)\right]+\lambda_{u i} \Delta U_{i}(k)^{T} \Delta U_{i}(k)
$$

With:

$$
Y_{C i}=\left[y_{C_{i}}\left(k+N_{i}+d_{i}\right) \cdots y_{C i}\left(k+H P_{i}+d_{i}\right)\right]
$$

The optimal control law is derived from analytical minimization of the previous cost function. Only the first control value is finally applied to the system.

$$
u_{i}(k)=u_{i}(k-1)+m_{G P C_{i}}^{T}\left[Y_{C i}(k)-\frac{\hat{G}(k) Y_{i}(k)+\hat{R} \Delta U_{i}(k-1)}{C_{i}\left(z^{-1}\right)}\right]
$$

With: $m_{G P C i}^{T}$ represents the first line of $\left(\hat{H}^{T} \chi_{i} \hat{H}+\lambda_{i i} I_{N_{G}}\right)^{-1} \hat{H}^{T}$ and $I_{N_{C}}$ is diagonal matrix of size $N_{C i} * N_{C i}$ and $\chi_{\mathrm{i}}$ is diagonal matrix of size $\mathrm{HP}_{\mathrm{i}} * \mathrm{HP}_{\mathrm{i}}$

$$
\chi_{\mathrm{i}}=\left(\begin{array}{ccc}
\lambda_{\mathrm{yi}} & & 0 \\
& \ddots & \\
0 & & \lambda_{\mathrm{yi}}
\end{array}\right) \mathrm{I}_{\mathrm{N}_{\mathrm{Ci}}}=\left(\begin{array}{lll}
1 & & 0 \\
& \ddots & \\
0 & & 1
\end{array}\right)
$$

We can express constraint on the process in the form:

$$
\begin{aligned}
& u_{\text {min }} \leq u_{i}(k) \leq u_{\max } \\
& -\Delta u_{s} \leq \Delta u_{i}(k) \leq \Delta u_{s}
\end{aligned}
$$

For the systems with constrains on the controller output value, on the controller increment output value or on the system output value, the vector $\Delta u$ is calculated by function FMINCON of Optimization Toolbox of the language Matlab.

D. Decoupling with Adjusting the weighting factor synchronization to the reference signal change

The main idea of decoupling controller is also described in the figure 7 .

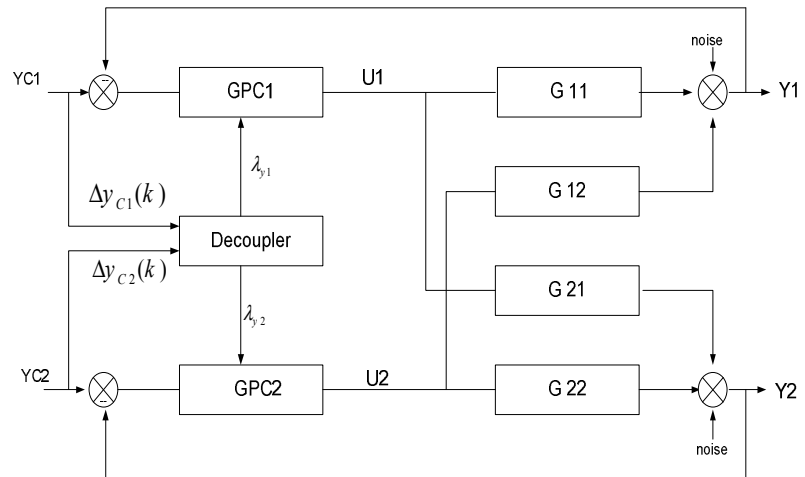

Fig. 7. Control structure of generalized predictive control with weighting factor adjusting.

We write the criterion $\mathrm{J}$ of each controller:

GPC1:

$$
\begin{aligned}
J_{l}= & \lambda_{y 1} \sum_{j=N_{l}}^{H P_{l}}\left[y_{C_{I}}(k+t)-\hat{y_{l}}(k+t)\right]^{2}+ \\
& \lambda_{u 1} \sum_{j=1}^{N_{C_{l}} \Delta u_{l}^{2}(k+t-1),}
\end{aligned}
$$

GPC2:

$$
\begin{aligned}
J_{2}= & \lambda_{y 2} \sum_{j=N_{2}}^{H P_{2}}\left[y_{C_{2}}(k+t)-\hat{y}_{2}(k+t)\right]^{2}+ \\
& \lambda_{u 2} \sum_{j=1}^{N_{C_{2}}} \Delta u_{2}^{2}(k+t-1)
\end{aligned}
$$

This control vector would minimize output deviation caused by reference changes [8]. Values $\lambda_{y i}$ are evaluated from equation:

$$
\lambda_{y i}=1+\sum_{j=1}^{m} \frac{K_{r j}}{M\left(q^{-1}\right)} \Delta y_{C j}(k)
$$

$K_{r j}:$ maximum error weight in $\mathrm{j}$ loop,

$\Delta y_{C j}:$ reference change on $\mathrm{j}$ input,

$M\left(q^{-1}\right)$ : polynomial of $q^{-1}$ defined by designer. 


\section{E. Decoupling with Adjusting the weighting factor synchronization by error observation}

To overcome the drawback of the previous method we present a new approach to decoupling the structure of the decoupling control is also described in the figure 8 .

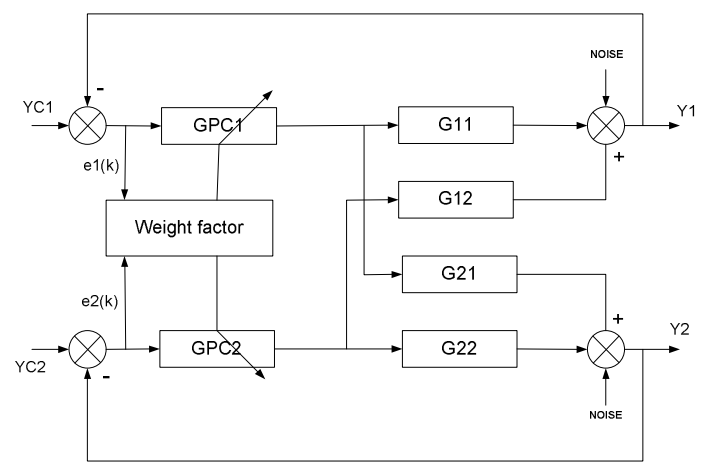

Fig. 8. Control structure of generalized predictive control with weighting factor adjusting.

The main idea of decoupling controller is the following: The error weight factor of the output variable whose reference is kept constant should be increased and synchronized with the variable whose reference is modified. We note that a high value of the weighting factor $\lambda_{\max }$ on output error can generate a very drastic change in controlled variables and degrades the control performance, what we are trying to avoid in most cases. On the other hand, a low value of weighting factor $\lambda_{\min }$ does not allow reducing the effect of the coupling caused by the other variable.

To satisfy some performance (smooth control movements and minimum coupling effect), we establish the weighting factors expressions

$$
\begin{aligned}
& \lambda_{y 1}=\left\{\begin{array}{cc}
\lambda_{\max 1} & \lambda_{y 1} \geq \lambda_{\max 1} \\
\lambda_{\min 1}+\exp \left(\left|\varepsilon_{2}(k)\right|\right) & \lambda_{y 1} \leq \lambda_{\max 1}
\end{array}\right\}, \\
& \lambda_{y 2}=\left\{\begin{array}{cc}
\lambda_{\max 2} & \lambda_{y 2} \geq \lambda_{\max 2} \\
\lambda_{\min 2}+\exp \left(\left|\varepsilon_{1}(k)\right|\right) & \lambda_{y 2} \leq \lambda_{\max 2}
\end{array}\right\} .
\end{aligned}
$$

With:

$$
\varepsilon_{1}(k)=g_{1}\left(y_{C_{1}}(k)-y_{1}(k)\right), \quad \varepsilon_{2}(k)=g_{2}\left(y_{C_{2}}(k)-y_{2}(k)\right) \text {. }
$$

\section{COMPUTER SIMULATIONS}

The described model (10) has strong interaction between input $u_{1}$ and output $y_{2}$. For this reason, the analysis has been done by changing temperature's reference and observing output signal of the humidity. The proposed method based on tuning the weighting factor $\lambda_{v 2}$ with error observation was applied to decoupling temperature and humidity outputs. Also, simulation results are compared with decoupling by adjusting the weighting factor $\lambda_{y 2}$ with the reference signal change, which proposed in literature. For $\lambda_{v 1}$, it remains constant and justified by the low interaction between $u_{2}$ and $G_{11}$. The principal parameters are set as follows: The prediction horizons are $H P_{1}=15, H P_{2}=15$, the control horizons $\operatorname{are}_{N c_{1}}=N c_{2}=1$ and the weighting factors of the control increments are $\lambda_{u 1}=9.4949 \mathrm{e}-4, \lambda_{u 2}=0.4712$.

A good decoupling could be achieved by the same dependence of the weighting factors as in equation (31) with:

$$
\lambda_{y 1}=1 \text { and } \lambda_{y 2}=\left\{\begin{array}{cc}
20 & \lambda_{y 2} \geq 20 \\
1+\exp \left(\left|\varepsilon_{1}(k)\right|\right) & \lambda_{y 2} \leq 20
\end{array}\right\}
$$

Simulation results obtained with this method are compared with decoupling by adjusting the weighting factor $\lambda_{y 2}$ with the reference signal change that proposed in literature:

$$
\lambda_{y l}=1,\left\{\begin{array}{l}
\lambda_{y 2}=1+\sum_{j=1}^{m} \frac{K_{r l}}{M\left(q^{-l}\right)} \Delta y_{C l}(k) \\
W i t h: \\
K_{r l}=2.5 \\
M\left(q^{-l}\right)=1-0.95 q^{-1}
\end{array}\right.
$$

The multivariable control is simulated with constraints and at sampling times $T=20$ seconds. The chosen input constraints are: $0 \% \leq u_{1} \leq 100 \%, 0 \% \leq u_{2} \leq 100 \%$.

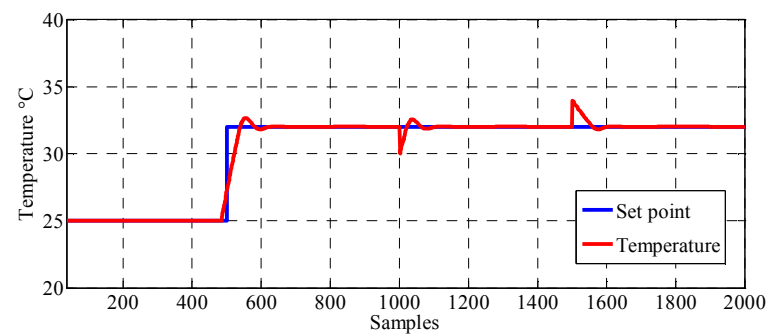

Fig. 9. Simulation result of set-point tracking temperature for predictive decoupling control.

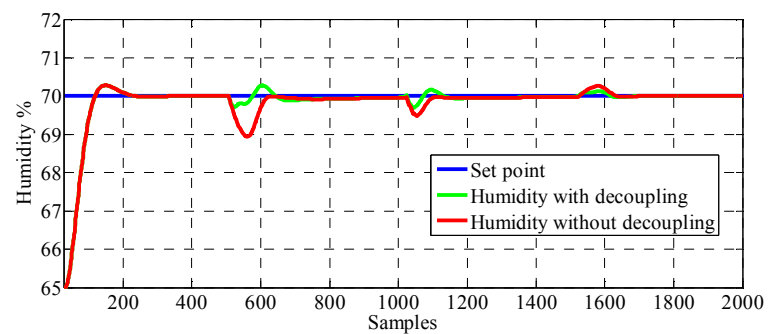

Fig. 10. Decoupling humidity with tuning weight factor by error output observation.

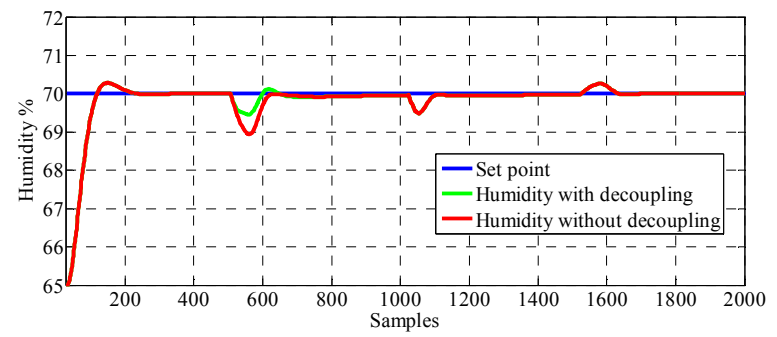

Fig. 11. Decoupling humidity with tuning weight factor by reference change 


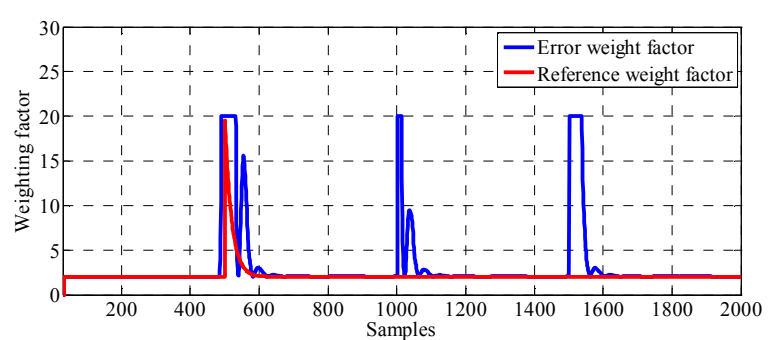

Fig. 12. Evolution of the weighting factor synchronized with change set point and with error weight factor observation.

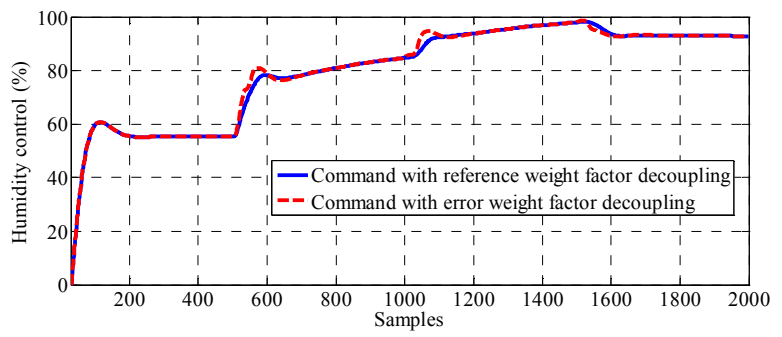

Fig. 13. Compared results of decoupling control humidity

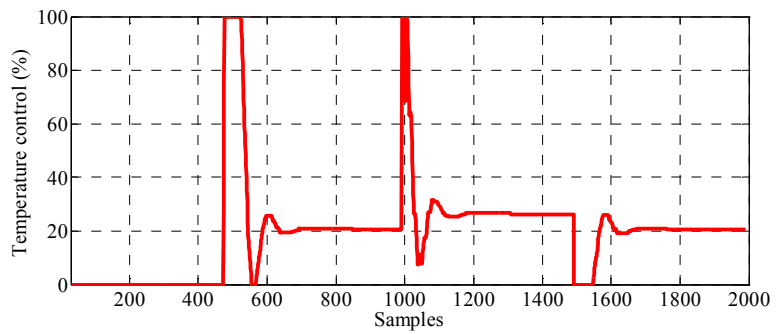

Fig. 14. Temperature control

The analysis has been done by changing temperature's reference and observing output signals of the humidity with and without decoupling. Figure 10 illustrates the simulation results using the proposed decoupled GPC control. After stationary had been reached, temperature reference increased at $\mathrm{k}=500$ from $25^{\circ} \mathrm{C}$ to $32^{\circ} \mathrm{C}$ and at $\mathrm{k}=1000$ to $\mathrm{k}=1500$, we added an external disturbance that is seen in Fig. 9. In Fig. 10, comparing the simulation result of set-point tracking humidity for predictive control with and without decoupling, it can be noticed that there are great differences in the R.H (Relative Humidity) response due to the proposed decoupling controller. However, in Fig.11 simulation results obtained with decoupling GPC that proposed in literature take care only of interactions caused by change of temperature reference. In Fig.12, we present the evolution of the weighting factor with error output and the evolution of the weighting factor which is synchronized with reference change. It is clear that tuning weighting factor with reference change does not reduce the effect of coupling due to external perturbation. But decoupling with tuning weight factor with error observation seems a good method to reduce the coupling effect due to the references change or external perturbation.

\section{CONCLUSION}

In this study, we have developed a decoupled GPC control, which takes into account natural constraints on the actors (the heater and nebulizer). Simulations results have been demonstrated that decoupling by tuning weighting factor with observation error is simpler for implementation than decoupling by adding interaction compensators and more effective than decoupling with weighting factor synchronized with change set point.

Main advantage of the weight decoupling method is its simplicity and computational burden, which makes it more suitable as adaptive controller for complex system than others decoupling method.

\section{REFERENCES}

[1] J.L. Costa, C.S. Freire, B.A. Silva, M.P. Cursino, R. Oliveira, A. M. Pereira and F.L. Silva, "Humidity control system in newborn incubator", Fundamental and Applied Metrology, 2009

[2] M. U. Cavalcante, B.C. Torrico, O. da Mota Almeida, A.P. de Souza Braga and F.L.M. da Costa Filho crossref, "Filtered Model-Based Predictive Control Applied to the Temperature and Humidity Control of a Neonatal Incubator ",9th IEEE/IAS International Conference on Industry Applications, 2010

[3] D. Bouattoura, P. Villon, and G. Farges, "Dynamic Programming Approach for Newborn's Incubator Humidity Control”, IEEE Transactions on Biomedical engineering, vol. 45, no. 1, 1998

[4] M. Hovd, and S. Skogestad, "Sequential Design of Decentralized Controllers." Automatica, vol. 30, pp. 1601-1607, 1994

[5] S.-J. Shiu, and S.-H. Hwang, "Sequential Design Method for Multivariable Decoupling and Multiloop PID Controllers." Ind. Eng. Chem. Process Des. Dev., vol. 37, pp. 107-119, 1998

[6] M.U.Cavalcante, B.C.Torrico, O. da Mota Almeida, A.P. de Souza Braga, F.L.M. da Costa Filho, "Filtered model-based predictive control applied to the temperature and humidity control of a neonatal incubator ", Industry Applications (INDUSCON), 2010 9th IEEE/IAS, pp 1-6, 2010

[7] M. S. Chiu, and Y. Arkun, "A Methodology for Sequential Design of Robust Decentralized Control Systems." Automatica, vol. 28, pp. $997-$ 1001,1992

[8] M. A. Zermani, E. Feki, A. Mami. "Multivariable Control Applied to Temperature and Humidity Case Study: Neonate incubator", MED-2012 Mediterranean Conference on Control and Automation. Systems engineering, control and modeling, in Barcelona, Espagne, July 3-6, 2012

[9] D. W. Clarke, C. Mohtadi and P. S. Tuffs, "Generalized Predictive Control - Part I", Automatica, vol. 23, p.p. 137-160, 1987

[10] Li, G., Stoten, D.P., Tu, J.-Y., "Model predictive control of dynamically substructured systems with application to a servohydraulically actuated mechanical plant", IET Control Theory Appl, vol. 4, (2), pp. 253-264, 2010

[11] Bego, O. Peric, N. I. Petrovic, "Decoupling multivariable GPC with reference observation", 10th Mediterranean Electrotechnical Conference , vol.2, pp.819-822, 2000

[12] M. A. Zermani, E. Feki, A. Mami, "Application of Genetic Algorithms in identification and control of a new system humidification inside a newborn incubator", International Conference on Communications, Computing and Control Applications, pp. 1-6, 2011

[13] D. W. Clarke, C. Mohtadi and P. S. Tuffs, "Generalized Predictive Control - Part II", Automatica, vol. 23, p.p. 149, 1987 